Prefitem

IMSC/93- 6

January, 1993

\title{
ON THE ROTATING CHARGED BLACK STRING SOLUTION
}

\author{
SWAPNA MAHAPATRA * \\ Institute of Mathematical Sciences, \\ C. I. T. Campus, Madras-600113, India
}

\begin{abstract}
A rotating charged black string solution in the low energy effective field theory describing five dimensional heterotic string theory is constructed. This solution is labelled by mass, electric charge, axion charge and angular momentum per unit length. The extremal limit of this solution is also studied.
\end{abstract}

^ e-mail: swapna@imsc.ernet.in 
The construction of classical solutions in string theory has received much attention in recent times $[1-7]$. The study of these solutions has shown the existence of black hole and extended black holes i.e. black strings and black p-brane type structures. Solvable conformal field theories are known in some cases [8]. These black holes and extended objects in string theory are very different from those which occur in general relativity because of the presence of the nontrivial dilaton field. In Einstein- Maxwell theory, all stationary black holes are described by the KerrNewman solutions parametrized by three quantities, namely the mass $M$, charge $Q$ and the angular momentum parameter $a$. When $a=0$, the solution describes the charged black hole solution or the Reissner-Nordstrom solution in general relativity. When $Q=0=a$, the solution reduces to that of the Schwarzschild solution. The vacuum Kerr family of solutions have $Q=0$ and they describe the rotating uncharged black hole solution. The generalizations of all these solutions to higher dimensions have also been discussed in the literature.

The study of these classical solutions in string theory is important as string theory is expected to provide us with a consistent quantum theory of gravity and also it will help us in understanding the basic nature of string theory itself. There exists a vast literature in this area of research and we shall not go into the details of it. For recent review, see ref.[9]. In this paper, we shall only concentrate on the rotating charged black string solution which can appear in the low energy heterotic string theory. Rotating charged black hole solutions in string theory have been obtained by Sen [10]. These solutions have been obtained from the Kerr solution in general relativity by using the method of twisting $[11-18]$. The method of twisting basically means that in string theory, if we have an exact classical solution which is independent of $d$ of the space-time coordinates, then we can perform an $0(d) \times 0(d)$ transformation on the solution, which produces new inequivalent classical solutions satisfying the same equation of motion derived from the low energy string effective action. Similarly in the case of heterotic string theory, the space of classical solutions which are independent of $d$ of the space- time directions and for which the gauge field configuration lies in a subgroup that commutes with $p$ 
of the $U(1)$ generators of the gauge group, has an $0(d) \times 0(d+p)$ or more generally $0(d-1,1) \times 0(d+p-1,1)$ symmetry and using this transformation, one can generate new inequivalent classical solutions starting from the known ones [13]. Various interesting solutions have been constructed by using this twisting method and the application of this solution has been widely studied [10-18]. The same philosophy has been used in ref.[10] to generate the rotating charged black hole solution in heterotic string theory starting from the Kerr solution.

It is also important to study the black string solution in order to have a deeper understanding of the string theory itself. Black strings are one dimensional extended objects surrounded by event horizons. There exists a rich variety of extended black hole solutions in ten dimensional string theory and they are closely related to the string soliton solution as well as to the fundamental strings [19]. These solutions are labelled by mass and axion charge per unit length. Rotating black string solutions have also been obtained by Horne and Horowitz [20], where the solution is parametrized by mass, axion charge, and angular momentum per unit length. However, they have not included the Maxwell field in the low energy effective action. In this paper, we construct the most general rotating charged black string solution carrying mass, electric charge, axion charge and angular momentum in the five dimensional low energy field theory describing heterotic string theory. Once again we use the method of twisting to obtain this solution starting from a four dimensional Kerr solution with an extra flat direction. We study the extremal limit of this solution to know the behaviour of extremal black string when both charge and rotation are present. We find that angular momentum dominates over the charge in the extremal limit which is also a characteristic feature of rotating charged black hole solution.

We start with the low energy action for heterotic string theory in five dimensions. Apart from the five dimensional string metric, we have dilaton, antisymmetric tensor gauge field and the Maxwell field. We do not consider the massless fields arising due to compactification and also we retain only terms with two or less number of derivatives in the action. Such an action is given by, 


$$
S=\int d^{5} x \sqrt{-g} e^{-2 \Phi}\left(-R-4(\nabla \Phi)^{2}+\frac{1}{12} H^{2}+\frac{1}{2} F^{2}\right)
$$

Here, $g_{\mu \nu}$ and $\Phi$ denote the metric and the dilaton field. $R$ is the five dimensional Ricci scalar and $F_{\mu \nu}=\partial_{\mu} A_{\nu}-\partial_{\nu} A_{\mu}$, is the field strength corresponding to the $U(1)$ gauge field $A_{\mu}$. The three form $H$ is given by,

$$
H_{\mu \nu \rho}=\partial_{\mu} B_{\mu \nu}+\text { cyclic permutations }-\left(\Omega_{3}(A)\right)_{\mu \nu \rho},
$$

where, $B_{\mu \nu}$ is the antisymmetric tensor gauge field and $\left(\Omega_{3}(A)\right)_{\mu \nu \rho}$ is the gauge Chern-Simons term. The Lorentz C-S term has been neglected as they involve more than two derivatives in the action.

Given a solution $G_{\mu \nu}, B_{\mu \nu}, \Phi$ and $A_{\mu}$, of the classical equation of motion, we would like to obtain the new transformed solution which also satisfies the same equations of motion derived from the action (1), by using the twisting procedure. For this purpose, we define an $11 \times 11$ matrix $\mathcal{M}$ as [13],

$$
\mathcal{M}=\left(\begin{array}{ccc}
\left(\mathcal{K}^{T}-\eta\right) g^{-1}(\mathcal{K}-\eta) & \left(\mathcal{K}^{T}-\eta\right) g^{-1}(\mathcal{K}+\eta) & -\left(\mathcal{K}^{T}-\eta\right) g^{-1} A \\
\left(\mathcal{K}^{T}+\eta\right) g^{-1}(\mathcal{K}-\eta) & \left(\mathcal{K}^{T}+\eta\right) g^{-1}(\mathcal{K}+\eta) & -\left(\mathcal{K}^{T}+\eta\right) g^{-1} A \\
-A^{T} g^{-1}(\mathcal{K}-\eta) & -A^{T} g^{-1}(\mathcal{K}+\eta) & A^{T} g^{-1} A
\end{array}\right)
$$

where,

$$
\mathcal{K}_{\mu \nu}=-B_{\mu \nu}-g_{\mu \nu}-\frac{1}{4} A_{\mu} A_{\nu}
$$

and, 


$$
\eta_{\mu \nu}=\operatorname{diag}(1,1,1,1,-1)
$$

Here, $g_{\mu \nu}$ and $B_{\mu \nu}$ are $5 \times 5$ matrices and $A_{\mu}$ is a five dimensional column vector. So given the solution of the equations of motion (as derived from action (1)), one can generate the new inequivalent solution, where the two solutions are related through the following relations:

$$
\mathcal{M}^{\prime}=\Omega \mathcal{M} \Omega^{T} ; \quad \Phi^{\prime}-\frac{1}{2} \ln \sqrt{\operatorname{det} g^{\prime}}=\Phi-\frac{1}{2} \ln \sqrt{\operatorname{det} g}
$$

where, $\mathcal{M}^{\prime}$ is the same matrix as $\mathcal{M}$ but with the new variables $g_{\mu \nu}^{\prime}, B_{\mu \nu}^{\prime}$ and $A_{\mu}^{\prime} . \Omega$ is an $O(5,6)$ matrix satisfying,

$$
\Omega L \Omega^{T}=L
$$

where,

$$
L=\operatorname{diag}\left(\eta_{5},-\eta_{6}\right)
$$

We want to generate the electrically charged rotating black string solution as an application of this transformation. Basically one starts with the four dimensional Kerr solution describing the rotating black hole and adds one extra dimension to it. The corresponding metric is given by,

$$
\begin{aligned}
d s^{2} & =-\frac{\left(\Delta-a^{2} \sin ^{2} \theta\right)}{\Sigma} d t^{2}-\frac{2 a \sin ^{2} \theta\left(r^{2}+a^{2}-\Delta\right)}{\Sigma} d t d \phi \\
& +\left[\frac{\left(r^{2}+a^{2}\right)^{2}-\Delta a^{2} \sin ^{2} \theta}{\Sigma}\right] \sin ^{2} \theta d \phi^{2}+\frac{\Sigma}{\Delta} d r^{2}+\Sigma d \theta^{2}+d x^{2}
\end{aligned}
$$

where, $\Delta=r^{2}+a^{2}-2 m r ; \Sigma=r^{2}+a^{2} \cos ^{2} \theta$; and $a$ is the angular momentum parameter. 
We also have,

$$
\Phi=0 ; \quad B_{\mu \nu}=0 ; \quad A_{\mu}=0
$$

In order to obtain the black string solution with non zero axion charge, one Lorentz boosts the solution to produce a non zero linear momentum along the $x$ (extra flat) direction and then uses the sigma model duality relations to convert this momentum to charge [21]. This is a novel way of adding axion charge to a static and translationally invariant solution. One knows that given a solution $g_{\mu \nu}, B_{\mu \nu}$ and $\Phi$ with a translational symmetry in $x$ direction, the dual solution is obtained from the following relations [22],

$$
\begin{aligned}
\tilde{g}_{x x} & =\frac{1}{g_{x x}} ; \quad \tilde{g}_{x \alpha}=\frac{B_{x \alpha}}{g_{x x}} ; \\
\tilde{g}_{\alpha \beta} & =g_{\alpha \beta}-\frac{\left(g_{x \alpha} g_{x \beta}-B_{x \alpha} B_{x \beta}\right)}{g_{x x}} ; \\
\tilde{B}_{x \alpha} & =\frac{g_{x \alpha}}{g_{x x}} ; \quad \tilde{B}_{\alpha \beta}=B_{\alpha \beta}-\frac{2 g_{x[\alpha} B_{\beta] x}}{g_{x x}} ; \\
\tilde{\Phi} & =\Phi+\frac{1}{2} \log g_{x x}
\end{aligned}
$$

In fact this was the approach taken by Horne and Horowitz to construct the rotating black string solution with a nonzero axion charge. Their solution is given by,

$$
\begin{aligned}
d s^{2} & =-\frac{(1-Z)}{B^{2}} d t^{2}-\frac{2 a Z \sin ^{2} \theta}{B^{2} \sqrt{1-v^{2}}} d t d \phi \\
& +\left[\left(r^{2}+a^{2}\right)+a^{2} \sin ^{2} \theta \frac{Z}{B^{2}}\right] \sin ^{2} \theta d \phi^{2}+\frac{\Sigma}{\Delta} d r^{2} \\
& +\Sigma d \theta^{2}+B^{-2} d x^{2},
\end{aligned}
$$

where, $Z=\frac{2 m r}{\Sigma}$. The dilaton and the non zero components of the antisymmetric tensor field are given by, 


$$
\begin{gathered}
\tilde{\Phi}=-\log B, \\
\tilde{B}_{x t}=\frac{v}{1-v^{2}} \frac{Z}{B^{2}} ; \quad \tilde{B}_{x \phi}=-\frac{a Z v \sin ^{2} \theta}{B^{2} \sqrt{1-v^{2}}}
\end{gathered}
$$

Here $v$ is the boost velocity. This solution is very much similar to the KaluzaKlein black hole, which was obtained for the dilaton coupling parameter $\alpha=$ $\sqrt{3}$ [23]. We can always perform an $O(d, d)$ transformation on this electrically neutral solution to obtain the charged rotating black string solution. But instead of doing this, we shall perform the $O(d, d)$ transformation on the four dimensional Kerr solution with an extra flat direction (eqn. 9), which will automatically generate the rotating black string solution with a nonzero electric as well as axionic charge. In order to obtain the inequivalent field configurations, we consider a mixing between the coordinates $t, x$ and one of the internal coordinate $y$ corresponding to one of the non abelian gauge field included in the action. So the most general transformed solution is obtained by first considering a boost in the $t-y$ ( $y$ is the coordinate in the internal space) direction, followed by a boost in the $t-x$ direction. The matrix $\Omega$ is given by,

$$
\Omega=\left(\begin{array}{llll}
I_{3} & & & \\
& S & & \\
& & I_{3} & \\
& & & R
\end{array}\right)
$$

where $S$ and $R$ are $O(1,1)$ and $O(2,1)$ matrices associated with the Lorentz transformation respectively. We choose the matrix $S$ to be identity matrix. The matrix $R$ is chosen to be, 


$$
R=\left(\begin{array}{ccc}
\cosh \alpha_{2} & \sinh \alpha_{2} & 0 \\
\sinh \alpha_{2} & \cosh \alpha_{2} & 0 \\
0 & 0 & 1
\end{array}\right)\left(\begin{array}{ccc}
\cosh \alpha_{1} & 0 & \sinh \alpha_{1} \\
0 & 1 & 0 \\
\sinh \alpha_{1} & 0 & \cosh \alpha_{1}
\end{array}\right)
$$

where, $\alpha_{1}$ and $\alpha_{2}$ are arbitrary parameters. So the matrix $\Omega$ is given by,

$$
\Omega=\left(\begin{array}{cccc}
I_{8} & & & \\
& \cosh \alpha_{1} \cosh \alpha_{2} & \sinh \alpha_{2} & \cosh \alpha_{2} \sinh \alpha_{1} \\
\sinh \alpha_{2} \cosh \alpha_{1} & \cosh \alpha_{2} & \sinh \alpha_{1} \sinh \alpha_{2} \\
\sinh \alpha_{1} & 0 & \cosh \alpha_{1}
\end{array}\right)
$$

With this choice of $\Omega$, we determine the transformed solutions from the relation, $\mathcal{M}^{\prime}=\Omega \mathcal{M} \Omega^{T}$. The transformed metric is given by,

$$
\begin{aligned}
d s^{\prime 2} & =-\frac{\left[\Sigma\left(\Delta-a^{2} \sin ^{2} \theta\right)-\beta^{2} m^{2} r^{2}\right]}{[\Sigma-m r(1-A)]^{2}} d t^{2}+\frac{2 \beta m r}{\Sigma-m r(1-A)} d x d t+\frac{2 \beta m r a \sin ^{2} \theta}{\Sigma-m r(1-A)} d x d \phi \\
& -\frac{2 m r a \sin ^{2} \theta\left[(1+A) \Sigma+\beta^{2} m r\right]}{[\Sigma-m r(1-A)]^{2}} d t d \phi+\left\{\left[\frac{\left(r^{2}+a^{2}\right)^{2}-\Delta a^{2} \sin ^{2} \theta}{\Sigma}\right] \sin ^{2} \theta\right. \\
& \left.+\frac{m^{2} r^{2} a^{2} \sin ^{4} \theta}{\Sigma}\left[\frac{2(1-A)}{\Sigma-m r(1-A)}-\frac{\gamma^{2} \Sigma}{(\Sigma-m r(1-A))^{2}}\right]\right\} d \phi^{2} \\
& +\frac{\Sigma}{\Delta} d r^{2}+\Sigma d \theta^{2}+d x^{2}
\end{aligned}
$$

The nonzero components of the gauge field and the antisymmetric tensor field are given by,

$$
\begin{aligned}
A_{t}^{\prime} & =\frac{2 m r \gamma}{\Sigma-m r(1-A)} ; \\
A_{\phi}^{\prime} & =\frac{-2 m r \gamma a \sin ^{2} \theta}{\Sigma-m r(1-A)} ; \\
A_{x}^{\prime} & =0
\end{aligned}
$$

and, 


$$
\begin{aligned}
B_{t x}^{\prime} & =\frac{\beta m r}{\Sigma-m r(1-A)} ; \\
B_{\phi x}^{\prime} & =\frac{-m r a \sin ^{2} \theta}{\Sigma-m r(1-A)} ; \\
B_{\phi t}^{\prime} & =\frac{m r a(1-A) \sin ^{2} \theta}{\Sigma-m r(1-A)}
\end{aligned}
$$

. Here,

$$
\beta=\sinh \alpha_{2} \cosh \alpha_{1} ; \quad \gamma=\sinh \alpha_{1} ; \quad A=\cosh \alpha_{1} \cosh \alpha_{2}
$$

satisfying the relation $A^{2}=1+\beta^{2}+\gamma^{2}$. The dilaton field is given by,

$$
\Phi^{\prime}=-\log \left[1-\frac{m r}{\Sigma}(1-A)\right]^{1 / 2}
$$

From the above expressions, we see that in the limit $\alpha_{1}, \alpha_{2} \rightarrow 0$, the solution reduces to that of the Kerr solution with a flat direction. In the limit $\alpha_{2} \rightarrow 0$, this solution reduces to that of the rotating charged black hole solution of Sen, with an extra flat direction and with a replacement of $\Phi \rightarrow 2 \Phi$. In the limit when $a \rightarrow 0$, this solution exactly matches with the black string solution obtained by Hassan and Sen when we compactify five of the flat coordinates [13]. The Einstein metric is obtained by multiplying expression (20) with $e^{-\frac{4}{3} \Phi^{\prime}}$, i.e. $g_{\mu \nu}^{\prime E}=e^{-\frac{4}{3} \Phi^{\prime}} g_{\mu \nu}^{\prime \Sigma}$. We shall not here give the complicated expression for the Einstein metric. This solution has both an event horizon and an inner horizon at $r_{ \pm}=m \pm \sqrt{m^{2}-a^{2}}$ respectively. The physical mass per unit length is computed by using the ADM mass formula and the expression is given by,

$$
M=\frac{m}{2}(1+A)
$$

The electric charge $Q$ and the magnetic moment $\mu$ are determined from the 
asymptotic form of $A_{t}$ and $A_{\phi}$ respectively and the corresponding expressions are given by,

$$
\begin{aligned}
& Q=2 m \sinh \alpha_{1} ; \\
& \mu=2 m a \sinh \alpha_{1} .
\end{aligned}
$$

The expression for the angular momentum $J$ is obtained by knowing the asymptotic form of the component $g_{t \phi}^{\prime}$ of the metric and is given by,

$$
J=\frac{1}{2} m a(1+A)
$$

The gyromagnetic ratio is obtained from the standard expression,

$$
g=\frac{2 \mu M}{Q J}=2
$$

For the rotating black string solution with only a nonzero axionic charge [20], the gyromagnetic ratio $g$ is $2-v^{2}$. So in the ultrarelativistic limit, when $v \rightarrow 1, g$ becomes equal to 1 . In the limit when $v \rightarrow 0$, the $g$ factor becomes equal to 2 .

The extremal limit of the solution corresponds to $m^{2}=a^{2}$, where both the horizons coincide, i.e. $r_{+}=r_{-}$. The angular velocity $\Omega$ at the horizon is given by,

$$
\begin{aligned}
\Omega & =-\left.\frac{g_{t \phi}^{\prime}}{g_{\phi \phi}^{\prime}}\right|_{r=r_{+}} \\
& =\frac{a}{2 m\left[m+\sqrt{m^{2}-a^{2}}\right]\left(\frac{1+A}{2}\right)}\left[1+\frac{\beta^{2}}{1+A}\right]
\end{aligned}
$$

In the limit, $\alpha_{1}, \alpha_{2} \rightarrow 0$, this reduces to that of the expression for the Kerr solution. In the limit $\alpha_{2} \rightarrow 0$, it reduces to that of the four dimensional rotating black hole solution of Sen. In the limit when $a \rightarrow 0$, the angular velocity goes to 
zero. In the extremal limit $\left(m^{2}=a^{2}\right), \Omega$ goes as $\frac{1}{2 a}$ along with quantities depending on $\alpha_{1}, \alpha_{2}$. Now if one considers the $a \rightarrow 0$ limit, we find that $\Omega$ diverges. This is also true for the electrically neutral rotating black string solution.

We also compute the surface gravity of the black string which is given by,

$$
\begin{aligned}
\kappa & =\left.\lim _{r \rightarrow r_{+}} \sqrt{g^{\prime r r}} \partial_{r} \sqrt{-g_{t t}^{\prime}}\right|_{\theta=0} \\
& =\frac{\sqrt{\left(m^{2}-a^{2}\right)}}{2 m\left[m+\sqrt{m^{2}-a^{2}}\right]\left(\frac{1+A}{2}\right)}\left[1+\frac{\beta^{2}}{1+A}\right]
\end{aligned}
$$

This expression shows that in the extremal limit, surface gravity goes to zero. In the limit $a \rightarrow 0$, surface gravity is proportional to $\frac{1}{4 m}$. Infact it is given by,

$$
\kappa=\frac{1}{4 m\left(\frac{1+A}{2}\right)}\left[1+\frac{\beta^{2}}{1+A}\right]
$$

In the limit when $\alpha_{1}, \alpha_{2} \rightarrow 0$, it reduces to that of the expression for the Kerr solution and when $\alpha_{2} \rightarrow 0$, it reduces to that of the four dimensional rotating black hole solution. The Hawking temperature can be calculated using the relation, $T=\frac{\kappa}{2 \pi}$. For the rotating black string, Hawking temperature goes to zero in the extremal limit unlike the nonrotating case, where it diverges in the extremal limit.

To summarize, in this paper we have constructed the most general electrically charged rotating black string solution in the five dimensional low energy heterotic string theory using the powerful method of twisting, which allows us to generate new nontrivial solutions from the known ones. We have also studied the extremal limit of this solution carrying mass, electric charge, axion charge and angular momentum per unit length. The angular momentum was found to dominate over the charge in the extremal limit. The extremal limit is basically independent of $Q$. The extremal nonrotating black string corresponds to the fundamental string itself and is boost invariant in the $x-t$ plane [19]. This was also shown to be true for the nonrotating charged black string [16]. Rotating black string is quite different 
and we do not know whether in the extremal limit it can be viewed as the field outside the fundamental string as we expect the solution for the fundamental string to be spherically symmetric. The study of black strings and p-branes is certainly interesting because of their close relationship with the string soliton solution as well as the fundamental strings themselves. Also, it has been shown in ref.[21] that the dual solutions describing the extremal black strings are equivalent to plane fronted waves, which means that the corresponding metric describes a string moving at the speed of light. One would like to ask similar questions in the case where both rotation and charge are present. The other question we would like to ask is regarding the stability of these solutions. A more detailed study of black strings and p-branes will hopefully shed some light on these issues.

Acknowledgement: I would like to thank Ashoke Sen and S. Fawad Hassan for many useful discussions.

\section{REFERENCES}

N1 G. Gibbons and K. Maeda, Nucl. Phys. B298 (1988) 741; D. Garfinkle, G. Horowitz and A. Strominger, Phys. Rev. D43 (1991) 3140; G. Horowitz and A. Strominger, Nucl. Phys. B360 (1991) 197; A. Shapere, S. Trivedi and F. Wilczek, Mod. Phys. Lett. A6 (1991) 2677. N2 S. Giddings and A. Strominger, Phys. Rev. Lett. 67 (1991) 2930. N3 R. C. Myers, Nucl. Phys. B289 (1987) 701; R. C. Myers and M. Perry, Ann. Phys. 172 (1986) 304; C. Callan, R. C. Myers and M. Perry, Nucl. Phys. B311 (1988) 673. N4 A. Dabholkar, G. Gibbons, J. Harvey anf F. R. Ruiz, Nucl. Phys. B340 (1990) 33. N5 C. Callan, J. Harvey and A. Strominger, Nucl. Phys. B359 (1991) 611. N6 M. Duff and J. Lu, Phys. Rev. Lett. 66 (1991) 1402; Nucl. Phys. B354 (1991) 141. N7 G. Mandal, A. M. Sengupta and S. Wadia, Mod. Phys. Lett. A6 (1991) 1685. N8 E. Witten Phys. Rev. D44 (1991) 314; C. Nappi and E. Witten, IASSNS-HEP-92-38; J. Horne and G. Horowitz, Nucl. Phys. B368 (1992) 444. N9 A. Sen, TIFR/TH/92-57 (hep-th/9210050); G. Horowitz, UCSBTH-92-32 (hep-th/9210119); J. Harvey and A. Strominger, 
EFI-92-41 (hep-th/9209055). N10 A. Sen, Phys. Rev. Lett. 69 (1992), 1006. N11 G. Veneziano, Phys. Lett. B265 (1991) 287; K. Meissner and G. Veneziano, Phys. Lett. B267 (1991) 33; M. Gasperini, J. Maharana and G. Veneziano, Phys. Lett. B272 (1991) 277, CERN-TH-6634/92 (hep-th/9209052). N12 A. Sen, Phys. Lett. B271 (1991) 295; ibid B274 (1991) 34. N13 S. F. Hassan and A. Sen, Nucl. Phys. B375 (1992) 103. N14 S. Kar, S. Khastgir and A. Kumar, Mod. Phys. Lett. A7 (1992) 1545; S. Khastgir and A. Kumar, Mod. Phys. Lett. A6 (1991) 3365; S. Kar and A. Kumar, IP-BBSR-92-18; J. Maharana, CALT-68-1781; S. Khastgir and J. Maharana, IP-BBSR-92-38 (hep-th/9206017); A. Kumar, Phys. Lett. B293 (1992) 49; P. Horava, Phys. Lett. B278 (1992) 101; J. Panvel, Phys. Lett. B284 (1992) 50; S. F. Hassan and A. Sen, TIFR/TH/92-61 (hep-th/9210121); S. Khastgir and J. Maharana, IP-BBSR-92-77 (Nov. 1992). N15 J. Maharana and J. Schwarz, CALT-68-1790. N16 A. Sen, TIFR/TH/92-29, hep-th/9206016. N17 A. Giveon and A. Pasquinucci, Phys. Lett. B294 (1992) 162; A. Giveon and M. Rocek, Nucl. Phys. B380 (1992) 128. N18 M. Rocek and E. Verlinde, Nucl. Phys. B373 (1992) 630. N19 G. Horowitz and A. strominger, Nucl. Phys. B360 (1991) 197. N20 J. Horne and Horowitz, Phys. Rev. D46 (1992) 1340. N21 J. Horne, G. Horowitz and A. Steif, Phys. Rev. Lett. 68 (1992) 568. N22 T. Busher, Phys. Lett. B201 (1988) 466; ibid B194 (1987) 59. N23 V. P. Frolov, A. I. Zelnikov and U. Bleyer, Ann. Phys. 44 (Leipzig), (1987) 371; G. Gibbons and D. Wiltshire, Ann. Phys. 167 (1986) 201; 176 (1987) 393(E). 